\title{
Radiative MRI Coil Design Using Parasitic Scatterers MRI Yagi
}

Sanchez-Heredia, Juan D.; Avendal, Johan; Bibic, Adnan; Lau, Buon Kiong

Published in:

IEEE Transactions on Antennas and Propagation

Link to article, DOI:

10.1109/TAP.2018.2794400

Publication date:

2018

Document Version

Peer reviewed version

Link back to DTU Orbit

Citation (APA):

Sanchez-Heredia, J. D., Avendal, J., Bibic, A., \& Lau, B. K. (2018). Radiative MRI Coil Design Using Parasitic Scatterers: MRI Yagi. IEEE Transactions on Antennas and Propagation, 66(3), 1570 - 1575.

https://doi.org/10.1109/TAP.2018.2794400

\section{General rights}

Copyright and moral rights for the publications made accessible in the public portal are retained by the authors and/or other copyright owners and it is a condition of accessing publications that users recognise and abide by the legal requirements associated with these rights.

- Users may download and print one copy of any publication from the public portal for the purpose of private study or research.

- You may not further distribute the material or use it for any profit-making activity or commercial gain

- You may freely distribute the URL identifying the publication in the public portal 


\section{Radiative MRI Coil Design Using Parasitic Scatterers: MRI Yagi}

Juan D. Sánchez-Heredia, Johan Avendal, Adnan Bibic and Buon Kiong Lau, Senior Member, IEEE

\begin{abstract}
Conventionally, radiofrequency (RF) coils used for magnetic resonance imaging (MRI) are electrically small and designed for nearfield operation. Therefore, existing antenna design techniques are mostly irrelevant for RF coils. However, the use of higher frequencies in ultrahigh field (UHF) MRI allows for antenna design techniques to be adapted to RF coil designs. This study proposes the use of parasitic scatterers to improve the performance of an existing 7T MRI coil called the single-sided adapted dipole (SSAD) antenna. The results reveal that scatterers arranged in a Yagi fashion can be applied to reduce local specific absorption rate (SAR) maxima of a reference SSAD by $40 \%$ with only a $6 \%$ decrease in the propagated $B_{1}{ }^{+}$field at the tissue depth of $15 \mathrm{~cm}$. The higher directivity of the proposed design also decreasing the coupling with additional elements, making this antenna suitable for use in high density arrays. These findings show the potential of parasitic scatterers as an effective method to improve the performance of existing radiative MRI coils.
\end{abstract}

Index Terms-Magnetic resonance imaging, electromagnetic propagation in absorbing media, specific absorption rate, YagiUda antennas.

\section{INTRODUCTION}

$\mathrm{U}$ LTRAHIGH field magnetic resonance imaging (UHF MRI) provides images of internal organs with far greater details than previously possible. However, UHF MRI of deep organs is complicated due to the use of relatively high frequency of the radiofrequency (RF) signal as well as the effect of much shorter signal wavelength in biological tissue. These factors can lead to destructive interference within the sample, causing field inhomogeneity and lower signal-to-noise (SNR) at deep locations of the body [1]. In addition, the RF power deposition is increased due to the use of a higher frequency, and specific absorption rate (SAR) levels become an important concern from a radiation exposure safety point of view [2].

Currently, the use of radiative RF coils is the most promising technique to overcome the low penetration depth of RF fields in UHF MRI [3]. This is because the higher operating frequency and electrically larger RF coil structure enable known antenna design techniques to be adapted to this

Manuscript received February 3, 2017. This work was supported in part by Crafoord Foundation under Grant No. 20150695.

J. D. Sanchez-Heredia was with the Department of Electrical and Information Technology, Lund University, 22363 Lund, Sweden. He is now with the Center for Hyperpolarization in Magnetic Resonance, Technical University of Denmark (DTU), 2800 Kgs. Lyngby, Denmark (e-mail: jdsanch@elektro.dtu.dk).

B. K. Lau is with the Department of Electrical and Information Technology, Lund University, 22363 Lund, Sweden. (e-mail: bkl@eit.lth.se).

J. Avendal is with Lite-On Mobile AB, Ideon Science Park, 22370 Lund, Sweden (e-mail: johan.avendal@liteonmobile.com).

A. Bibic is with Lund University Bioimaging Center, Faculty of Medicine, Lund University, 22185 Lund, Sweden (e-mail: adnan.bibic@med.lu.se) application. One example of radiative coils is the Single-Sided Adapted Dipole (SSAD) concept [3], which consists of a halfwave dipole antenna placed on a rectangular block of highpermittivity substrate. Moreover, the field inhomogeneity of UHF MRI can be addressed through the use of coil arrays that employ appropriate phase weightings of the elements [4], and a 32-element coil array has been proposed [5]. Therefore, one important challenge of UHF MRI is to design radiative RF coils that provide improved penetration depth and are small enough to facilitate high density arrays. Radiative coil arrays are also unique due to their inherently low mutual coupling, in contrast to traditional loop arrays where the elements typically overlap and strongly couple to one another [6].

In this paper, we propose a new RF coil design strategy that greatly reduces the local SAR at the interface between coil and sample. The design concept (called MRI Yagi) is based on the well-known Yagi (or Yagi-Uda) antenna [7], [8] in that it exploits parasitic scatterers to modify some characteristics of the antenna radiation patterns. However, the proposed design takes the idea further by utilizing the scatterers to also reduce the electric nearfield, and hence the local SAR.

Parasitic scatterers have also been successfully used to provide decoupling between different active antennas [9]. By a proper choice of parameters, perfect matching and decoupling of the individual elements of an array can be obtained for a given element spacing without increasing the overall array size or introducing additional decoupling networks. As an example, Yan et al. applied this method to improve the decoupling of a monopole array used for 7T MRI [10].

The design proposed here provides a $25 \%$ improvement on the ratio of $B_{1}{ }^{+}$to local SAR, relative to an SSAD design of the same size [3]. Similar performance gain has also been achieved recently using a fractionated dipole antenna by Raaijmakers et al. [11]. However, the antenna proposed here is about half the size of the antenna in [11], which will allow twice as many elements to be implemented in a coil array of a given size. Moreover, whereas the fractionated dipole generates a lower $B_{1}{ }^{+}$than an SSAD close to its surface, the MRI Yagi does not suffer from this effect and it even provides a slightly higher $B_{1}{ }^{+}$at distances close to the coil $(<6 \mathrm{~cm})$.

\section{REFERENCE AND PROPOSED RF COILS}

\section{A. Reference Single-Side Adapted Dipole (SSAD)}

Following the design proposed by Raijmakers et al. [3], an SSAD was modeled using the time-domain solver of CST Microwave Studio. Distilled water (dielectric constant $\varepsilon_{r}=78$, conductivity $\left.\sigma=5.55 \times 10^{-6}[\mathrm{~S} / \mathrm{m}]\right)$ in a $1 \mathrm{~mm}$ thick clear plastic frame (PMMA, $\varepsilon_{r}=2.8$ and loss tangent $\tan \delta=0.02$ ) was used as the dielectric material to simplify the fabrication and validation process. The sample was modeled as a homogeneous rectangular phantom $\left(400 \times 400 \times 200 \mathrm{~mm}^{3}\right)$ with material properties representing average tissue properties at the operating frequency of $298.2 \mathrm{MHz}\left(\varepsilon_{r}=34, \sigma=0.4\right.$ $\mathrm{S} / \mathrm{m})$. The dipole was modeled as two PEC cylindrical wires (length of $45 \mathrm{~mm}$ and diameter of $1.6 \mathrm{~mm}$ ), separated by a 2 mm gap. 
After a first simulation using dimensions similar to those in [3], an optimization of the dimensions was performed in order to find the best compromise between maximizing the $B_{1}{ }^{+}$field at the depth of $15 \mathrm{~cm}$ and minimizing the local SAR (1g averaged). The final dimensions used for the SSAD are $162 \times$ $70 \times 50 \mathrm{~mm}^{3}$. All the simulations in this part of the study were normalized to $1 \mathrm{~W}$ of available power. A depth of $15 \mathrm{~cm}$ is significant for the imaging of deep organs (e.g., prostate and deep brain). $B_{1}{ }^{+}$is the left circularly polarized transverse magnetic field $\left(B_{1 x}+B_{1 y}\right) / 2$, assuming the main magnetic field $B_{0}$ along the positive $\mathrm{z}$ direction. $B_{1 x}$ and $B_{1 y}$ are the $\mathrm{x}-$ and $\mathrm{y}-$ component of the $B_{1}$ field due to the RF coil, respectively. It is noted that even RF coils that are not predominantly circularly polarized can offer a high $B_{1}{ }^{+}$field, if one of the field components $\left(B_{1 x}\right.$ or $B_{1 y}$ ) is high. Therefore, RF coils based on single-polarized linear dipoles are still useful for tipping the magnetic moments of the nuclear spins in an MRI experiment.

\section{B. Proposed RF Coil}

The proposed ("MRI Yagi") coil is constructed by adding parasitic scatterers (reflectors and directors) to the reference SSAD. The design concept is based on two key aspects:

- Use of reflectors to increase the directivity of the coil, reducing the back-propagation and hence the interaction of the coil with the rest of the scanner system.

- Use of directors in the main direction of propagation to modify the electric field (E-field) distribution in the close vicinity of the coil, mitigating E-field peaks which can lead to local SAR peaks.

The basic design rules for parasitic scatterers [7] are that reflectors have to be longer than the active element and placed at a distance of a quarter wavelength $(\lambda / 4)$ behind it, whereas directors should be shorter and be placed slightly further away than $\lambda / 4$ in front of it. These rules assume that the whole system formed by the dipole and the scatterers is placed in the same medium. However, placing a dipole over a highpermittivity dielectric substrate inherently improves the directivity. This is because the wave impedance of air $\left(Z_{0}\right)$ is higher than that of the high-permittivity medium $\left(Z_{d}\right)$ (since $Z_{d}$ $\approx Z_{0} / \sqrt{\varepsilon}_{r}$ ), resulting in the dielectric substrate being the preferred direction for the propagated wave from the dipole. To mitigate multiple reflections of the propagated wave, it is important to minimize the distance between the dielectric material and the sample (ideally they should be in contact). For this reason, the thickness of the PMMA bottom layer of the frame was designed to be as thin as possible $(1 \mathrm{~mm})$.

The final design as shown in Fig. 1 has the same overall size as the $\operatorname{SSAD}\left(162 \times 70 \times 50 \mathrm{~mm}^{3}\right)$. This was achieved by keeping the reflectors on the same plane as the dipole, which violated the rule of $\lambda / 4$ separation but was found to give a minor effect on the propagated $B_{1}{ }^{+}$field (see Section III-A). The detailed dimensions of this coil are given in Section III.

\section{PARAMETRIC STUDY}

\section{A. Effect of Reflector Position}

First, simulations with only the reflectors added to the

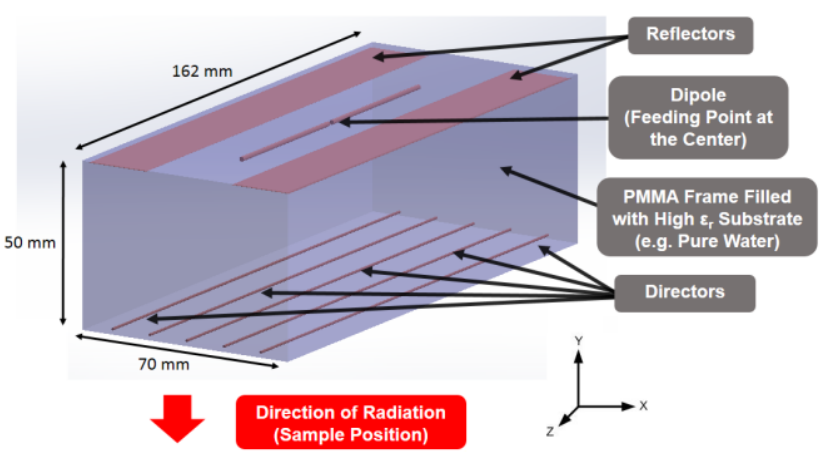

Fig. 1. Schematic of the proposed design using parasitic scatterers. Two reflectors and five directors are combined with an active element (dipole), all immersed inside a high $\varepsilon_{r}$ substrate (distilled water). The reference SSAD is identical in design but does not contain the reflectors and directors.

TABLE I

Simulated $B_{1}^{+}{ }_{\text {Front }} / B_{1}^{+}{ }_{\text {Back }}$ FOR DifFERENT REFLECTOR POSITIONS

\begin{tabular}{lccc}
\hline \hline \multicolumn{1}{c}{ Configuration } & $\begin{array}{c}B_{1}{ }^{+} \text {Front } \\
{[\mathrm{T}]}\end{array}$ & $\begin{array}{c}B_{1}{ }^{+} \text {Back } \\
{[\mathrm{T}]}\end{array}$ & $B_{1}{ }^{+}$Front $/ B_{1}{ }^{+}{ }_{\text {Back }}$ \\
\hline SSAD (without reflectors) & $7.02 \cdot 10^{-8}$ & $8.03 \cdot 10^{-8}$ & 0.87 \\
SSAD + Separated Reflectors & $7.52 \cdot 10^{-8}$ & $5.20 \cdot 10^{-8}$ & 1.44 \\
SSAD + On-Plane Reflectors & $7.26 \cdot 10^{-8}$ & $7.81 \cdot 10^{-8}$ & 0.93 \\
\hline \hline
\end{tabular}

$\overline{B_{1}{ }^{+} \text {Front }}=$ front propagated $B_{1}{ }^{+}$, at $15 \mathrm{~cm}$ deep into the phantom.

$B_{1}{ }^{+}$Back $=$back propagated $B_{1}{ }^{+}$, at $10 \mathrm{~cm}$ distance away from the coil edge.

reference SSAD were performed. The reflectors were modeled as two PEC thin sheets of dimensions $20 \times 162 \mathrm{~mm}^{2}$ with edge-to-edge separation of $30 \mathrm{~mm}$ (see the reflectors in Fig. 1). Wide reflectors are more effective in reducing backpropagation than narrower ones. Moreover, when they are placed coplanar with the dipole (as shown in Fig. 1), they should not be too close to the dipole, to avoid distorting the field distribution generated by the dipole. Two cases were studied: one where the plane containing the reflectors was separated by $\lambda / 4$ from the dipole (i.e., extending the substrate thickness of $50 \mathrm{~mm}$ by $\lambda / 4$ and moving the dipole into the substrate by $\lambda / 4$, along the direction of negative $y$-axis) and one where both the dipole and the reflectors were placed on the same plane (at the substrate surface), which corresponds to design in Fig. 1 but without the directors. The results were compared to the reference SSAD and summarized in Table I.

As can be seen in Table I, the addition of the reflectors significantly improved the ratio between propagated and backpropagated $B_{1}^{+}$relative to the reference SSAD. However, separating the reflectors from the plane containing the dipole by $\lambda / 4$ provided only a modest increase of $3.6 \%$ in the frontpropagated $\mathrm{B}_{1}{ }^{+}$. For this proof-of-concept, we consider that it is more important to keep the coil size small, and therefore the reflectors are kept on the same plane as the dipole. For applications where back-propagation is an issue, some separation should be considered.

\section{B. Position and Number of Directors}

Next, simulations with the directors added to the basic model of Section III-A (formed by the dipole, dielectric material and two reflectors) were performed in order to reduce the local SAR. Placing the scatterers inside the dielectric (bottom side in Fig. 1), has a strong effect on the E-Field 


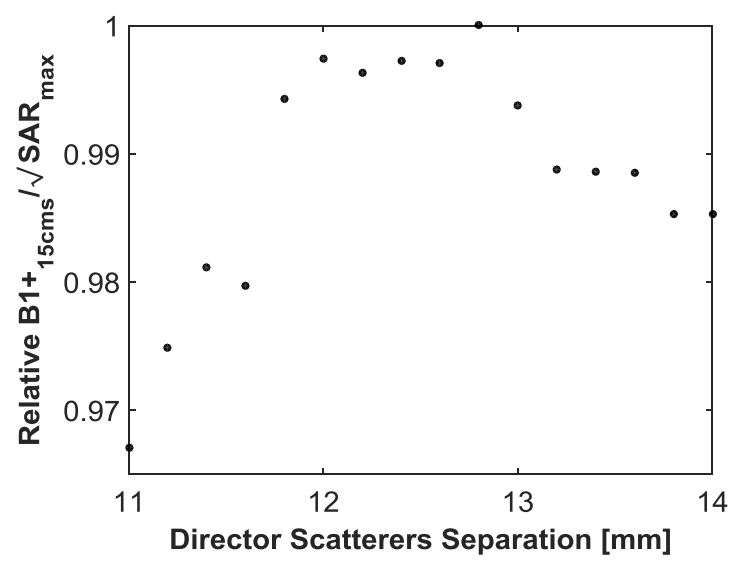

Fig. 2. Simulated $B_{1}^{+} / \sqrt{ } \mathrm{SAR}_{\mathrm{MAX}}$ for an MRI Yagi with different director separation.

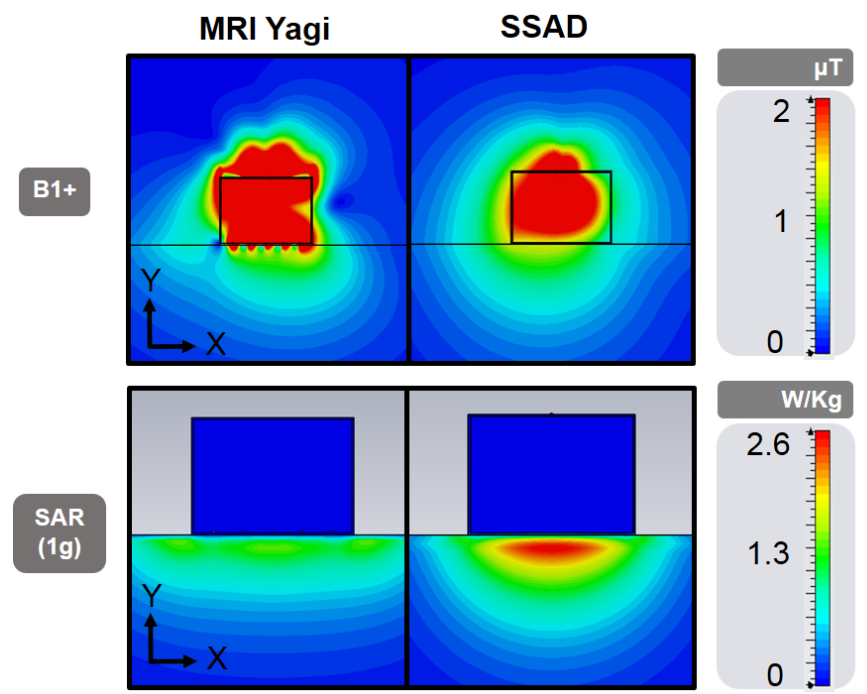

Fig. 3. Simulated $B_{1}{ }^{+}$and SAR (1g averaged) distributions of the MRI Yagi and the reference SSAD (normalized to $1 \mathrm{~W}$ available power) for the $x-y$ cut at the center of the coil. The coils are placed on a phantom of $400 \times 400 \times 200$ $\mathrm{mm}^{3}$, filled with material emulating tissue properties.

distribution inside the dielectric material. The strategy here was to add a layer of thin directors at the interface between the coil and the sample. This layer of directors modifies the phase distribution of the E-field around the scatterers and therefore its layout can be optimized to minimize local SAR. Keeping the directors thin enables them to modify the phase of the Efield without shielding the $\mathrm{H}$-field, and therefore allowing a propagated field towards the sample.

To keep the design simple, a single layer of thin directors was used with the sole purpose of reducing the local SAR while leaving the propagated $B_{1}{ }^{+}$relatively unaffected. The length of the directors was chosen to be equal to the coil length $(162 \mathrm{~mm})$, because when this length is reduced (e.g., to $100 \mathrm{~mm}$ ), local SAR maxima tend to form around the two end elements of the directors. It is noted that other parameters such as the number of director layers and their length (relative to each other) can also be varied to further optimize $B_{1}{ }^{+}$and local $\mathrm{SAR}$, but these detailed aspects are beyond the scope of this proof of concept study.

With this in mind, a parametric simulation was performed

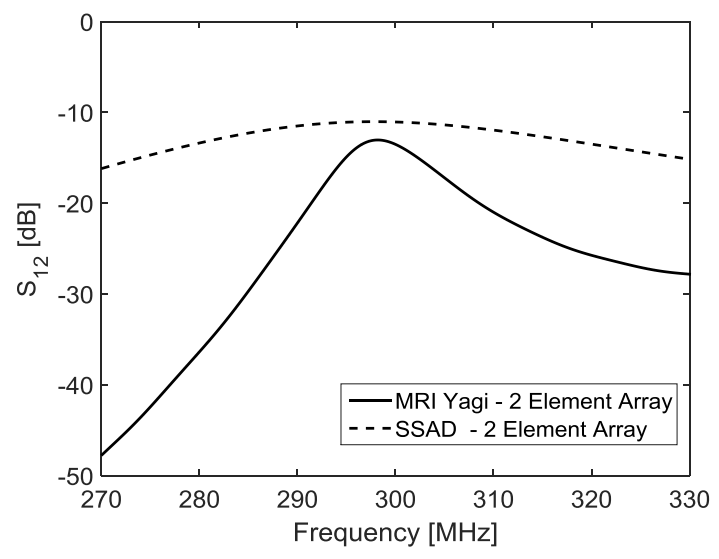

Fig. 4. Simulated $\mathrm{S}_{12}$ of two-element arrays with $30 \mathrm{~mm}$ element separation.

by varying the director separation. The results of $B_{1}{ }^{+}$ $/ \sqrt{ } \mathrm{SAR}_{\mathrm{MAX}}$ are shown in Fig. 2, where the $B_{1}{ }^{+}$field was sampled at a depth of $15 \mathrm{~cm}$ inside the phantom and SAR $\mathrm{MAX}$ is the maximum SAR ( $1 \mathrm{~g}$ averaged) over the whole sample.

It can be observed that the optimal relation between propagated $B_{1}{ }^{+}$and maximum local SAR is obtained when adjacent directors are separated by between 12 and $13 \mathrm{~mm}$, which is equivalent to $1 / 10$ to $1 / 8$ of the wavelength inside the dielectric. Therefore, a distance of $12.5 \mathrm{~mm}$ was chosen as the separation between the directors. Due to the presence of the parasitic scatterers changing the load condition, the length of the active dipole in the final MRI Yagi coil was increased to $101 \mathrm{~mm}$ (two dipole arms of $49 \mathrm{~mm}$ with a $2 \mathrm{~mm}$ feeding gap) to retune the coil.

The simulated $B_{1}{ }^{+}$and SAR distributions for the final coil design including all the scatterers are shown in Fig. 3, together with corresponding results from the reference SSAD. It can be seen from the SAR plots that the added directors effectively prevent the formation of the local maximum at the sample-tocoil interface that would otherwise occur in an SSAD. The maximum SAR is reduced by about $40 \%$ for the MRI Yagi ( $1.6 \mathrm{vs} 2.6 \mathrm{~W} / \mathrm{kg}$ ), while the propagated $B_{1}{ }^{+}$at $15 \mathrm{~cm}$ depth is only slightly reduced for the MRI Yagi (0.066 vs. $0.069 \mu \mathrm{T})$, which gives an overall $\approx 25 \%$ increase of the $B_{1}{ }^{+} / \sqrt{ } \mathrm{SAR}_{\mathrm{MAX}}$ ratio.

It is noted that the asymmetry of the $B_{1}{ }^{+}$distribution in Fig. 3 is due to the electric currents induced in the conductive elements (scatterers and sample) by the changing magnetic fields (H-fields). These currents generate secondary magnetic fields which are out of phase from the primary one generated by the dipole [12].

\section{Array Configuration}

The suitability of the proposed MRI Yagi coil design for array application was also evaluated. To this end, an array with two elements along the $x$-axis was simulated. The orientation of each element follows that of Fig. 1. The side-toside element separation was chosen to be as small as $0.03 \lambda$ (i.e., $30 \mathrm{~mm}$ ). The simulated mutual coupling was compared to a similar two-element array setup using the reference SSAD, and the results are shown in Fig. 4. The MRI Yagi array shows a coupling coefficient $\left(S_{12}\right)$ of $-14 \mathrm{~dB}$ at the resonance 


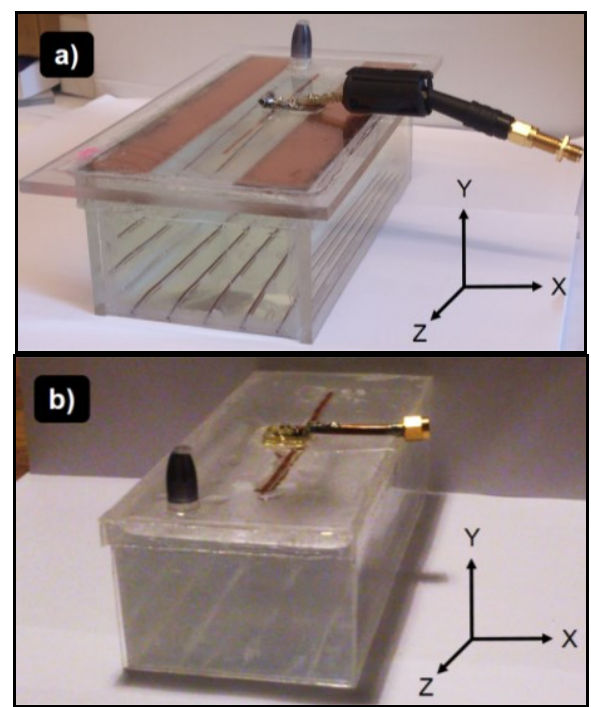

Fig. 5. Fabricated prototypes: a) MRI Yagi, b) SSAD.

frequency, which is about $3 \mathrm{~dB}$ better than the SSAD array. This result further confirms the improved directivity of the coil, which mainly results from the use of reflectors. The improved coupling performance can also be traded against smaller element separation to reduce the coil array size.

\section{VALIDATION}

\section{A. Prototype Fabrication}

To validate the simulation results, the proposed MRI Yagi and the reference SSAD were fabricated (see Fig. 5). As mentioned, the dielectric used for the coils was distilled water. Therefore, a water-tight frame was made for both coils using clear plastic (PMMA, $\varepsilon_{r}=2.8$ and $\tan \delta=0.02$ ) sheets from Evonik GmbH, Germany. For the SSAD, $1 \mathrm{~mm}$ thick PMMA sheets were used to construct the frame. For the MRI Yagi coil, the PMMA thickness used for the bottom side of the antennas was $1 \mathrm{~mm}$ to keep the distance to the sample as small as possible (similar to the SSAD), whereas thicker sheets (4 $\mathrm{mm}$ ) were used for the lateral and top sides to give better mechanical strength. Though the $4 \mathrm{~mm}$ thick sides are larger than the corresponding $1 \mathrm{~mm}$ thick sides in the simulation model, the increased thickness is not critical to the coil design due to the much higher permittivity of the distilled water as compared to both air and PMMA. The dipole arms were made with $1.6 \mathrm{~mm}$ thick cylindrical copper wires, glued to the inner side of the PMMA container to ensure good contact with the water. For the MRI Yagi, the reflectors were made using two copper tape strips (thickness of $0.07 \mathrm{~mm}$ ) attached to the upper-inner part of the PMMA frame, whereas the directors were implemented using $0.8 \mathrm{~mm}$ thick cylindrical copper wire attached to the bottom-inner part of the frame. All the copper wires used had a thin insulation coating to avoid oxidation.

The dipoles are fed through a small tuning and matching board placed on top of the coil's PMMA frame (outside the water) and then connected to a coax cable. Two 3-10pF trimmers (Sprague Goodman, SGC3S100NM) were added to each coil, one for tuning and one for matching, so that the coils could be finely tuned and matched to $50 \Omega$ for each of the loading conditions used during the experiments. For simplicity, a balun was not included in these tests, and instead ferrites were attached to the coaxial cable to prevent common mode currents on the cable shield.

\section{B. Near-Field Characterization in Free Space}

The fabricated coils were first characterized in terms of their near-field E-field and $\mathrm{H}$-field in free space at $298.2 \mathrm{MHz}$, using a probe calibrated for free space. The measurements were performed using a dosimetric DASY-6 system (Schmid \& Partner Engineering AG, Switzerland) located at Lite-On Mobile AB, Lund, Sweden. The coils were fed with a continuous wave with $250 \mathrm{~mW}$ power. The measurements were performed over an area of $170 \mathrm{~mm} \times 80 \mathrm{~mm}(0.5 \mathrm{~mm}$ resolution) along the $x z$-plane, $3 \mathrm{~mm}$ away from the bottom of the phantom and centered at the antenna center.

Although the free-space measurement is not representative of the real operating environment of the coils, these

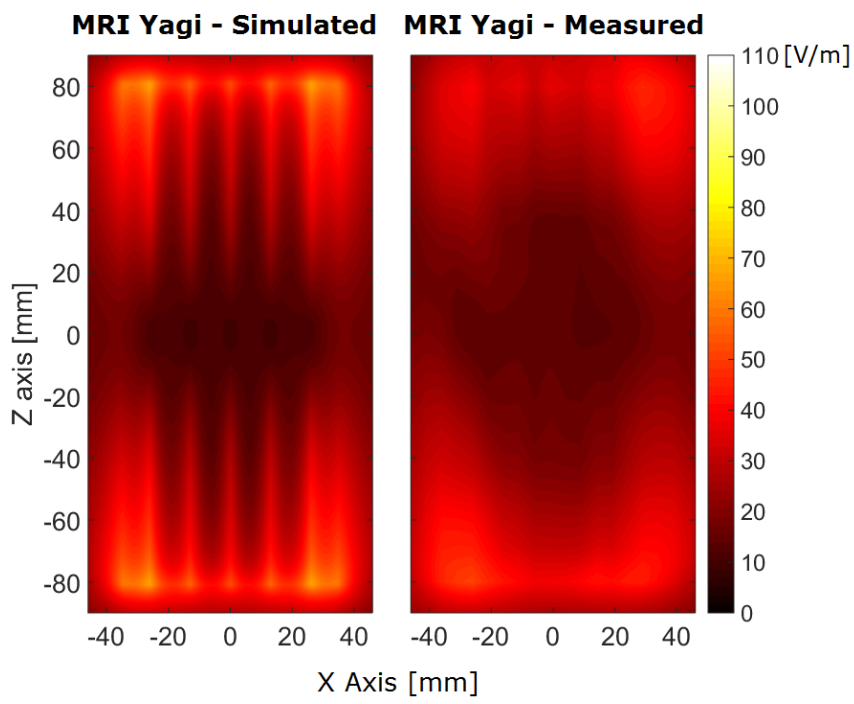

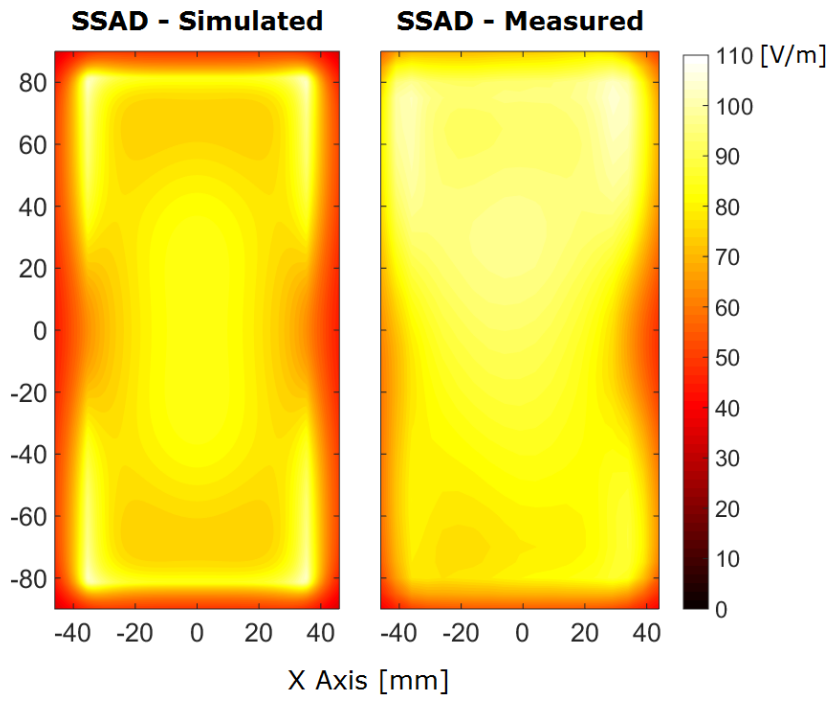

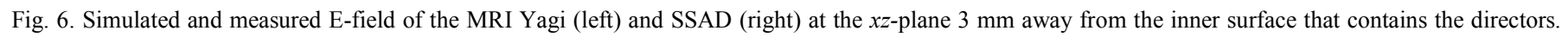
All measurements and simulations were performed with $250 \mathrm{~mW}$ input power, and in free space (no phantom was included in this section). 


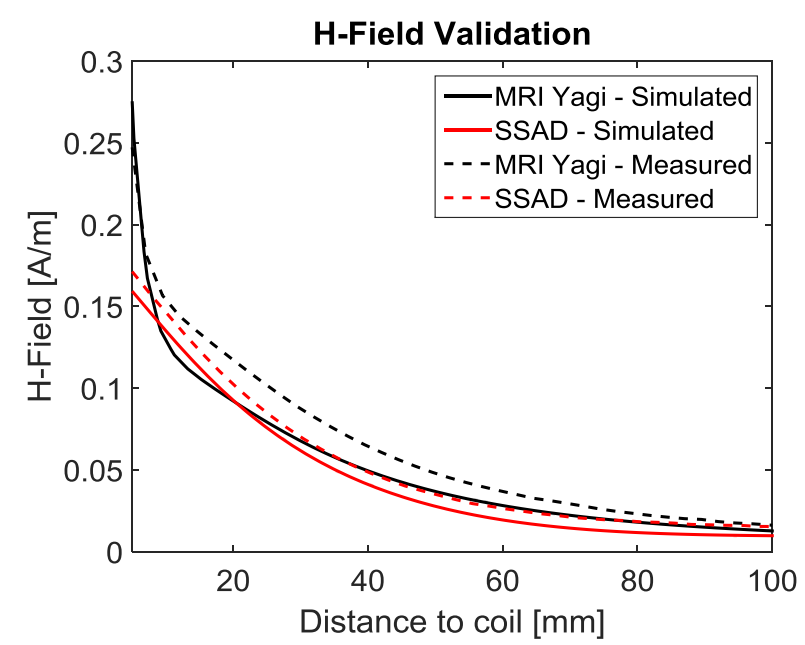

Fig. 7. Measured and simulated H-Field along the line defined by the coil center and the propagation direction.

measurements are nevertheless useful to determine the accuracy of the simulations and validate the E-field and $\mathrm{H}$ field distributions around the coils. Therefore, simulations of the two coils surrounded by free space (without phantom) were repeated for comparison with the measured near-field field distributions. Further, the free-space simulation results were obtained with models of the two coils that include the PMMA frame. As in the measurement, the simulated input power was also set to $250 \mathrm{~mW}$.

Two measurements were performed: the first one was to obtain the E-field over the $x y$-plane close to the coil surface (3 $\mathrm{mm}$ distance from the coil surface). The second one was to obtain the $\mathrm{H}$-field along the direction of positive $y$-axis, starting from the middle point of the coil surface with the directors. The reason of these choices is to prove that the Efield local maxima are indeed reduced at close vicinity of the MRI Yagi (when compared to the SSAD), while still allowing efficient propagated $\mathrm{H}$-field at a certain distance from the coil. The measured and simulated results for both coils are shown in Fig. 6 (E-field) and Fig. 7 (H-field). The measured E-field

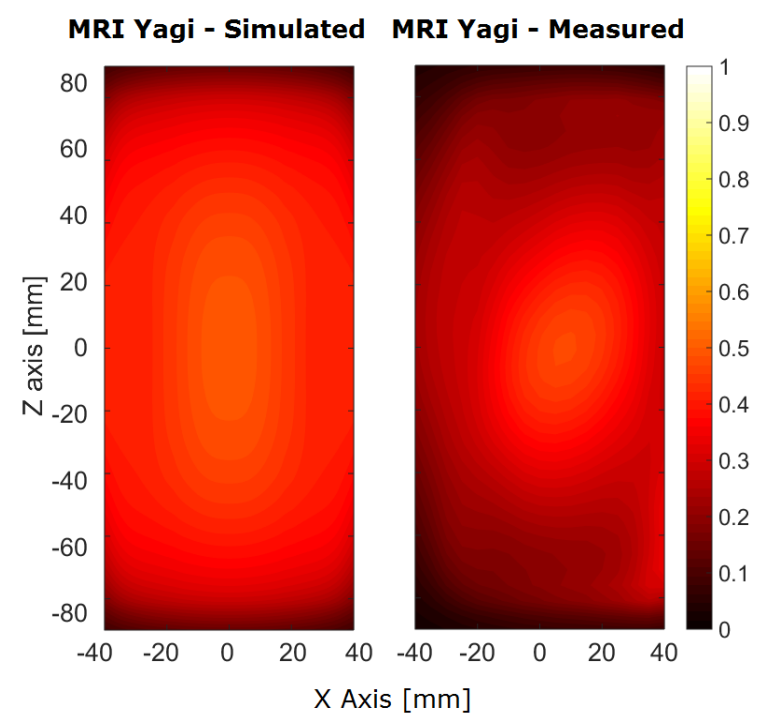

maximum for the MRI Yagi is $51.23 \mathrm{~V} / \mathrm{m}$, in comparison to $102.82 \mathrm{~V} / \mathrm{m}$ for the SSAD. These values agree well with the simulated ones ( 66.5 and $104.56 \mathrm{~V} / \mathrm{m}$ respectively). The Hfield measured along the positive $y$-axis in Fig. 7 also shows a reasonable agreement with the simulated results. It is observed from this measurement that at far distance from the coil, both prototypes show very similar $\mathrm{H}$-field. This result proves that the directors, which are used to reduce the localized E-field, do not prevent efficient wave propagation towards the sample.

\section{SAR Characterization with Sample}

To validate the performance of the MRI Yagi under realistic sample loading conditions, a SAR measurement was performed. Since the SAR probe was calibrated from around $700 \mathrm{MHz}$, only a relative measurement could be performed for the MRI Yagi and the reference SSAD (normalized to the maximum local SAR value measured for the SSAD). A SAM twin phantom (Schmid \& Partner Engineering AG, Zurich, Switzerland) filled with TSL-900 gel $\left(\varepsilon_{r}=49, \sigma=0.37\right.$, measured at $300 \mathrm{MHz}$ ) was used for this measurement. The measurement was performed over an area of $170 \mathrm{~mm} \times 80 \mathrm{~mm}$ ( $0.5 \mathrm{~mm}$ resolution) along the xz-plane, $3 \mathrm{~mm}$ away from the bottom of the phantom and centered at the antenna center. The SAM twin phantom is made of fiberglass $\left(\varepsilon_{r}=4.4\right)$, and its flat part has a thickness of $2 \mathrm{~mm}$.

Full-wave simulations of both antennas were also performed for the same setup, which includes the $2 \mathrm{~mm}$ fiberglass layer of the phantom and the electrical properties of TSL-900 at $300 \mathrm{MHz}$. The measured and simulated results are shown side by side for each antenna in Fig. 8. As can be seen, the maximum local SAR the MRI Yagi is about $50 \%$ lower than that of the SSAD. Good agreement is also achieved between the simulated and measured results.

\section{DISCUSSION}

Through the use of parasitic scatterers, the ratio $B_{1}^{+}$ $/{ } \mathrm{SAR}_{\mathrm{MAX}}$ can be improved for radiative MRI coils at deep locations in the sample. Reflectors are used to reduce back-

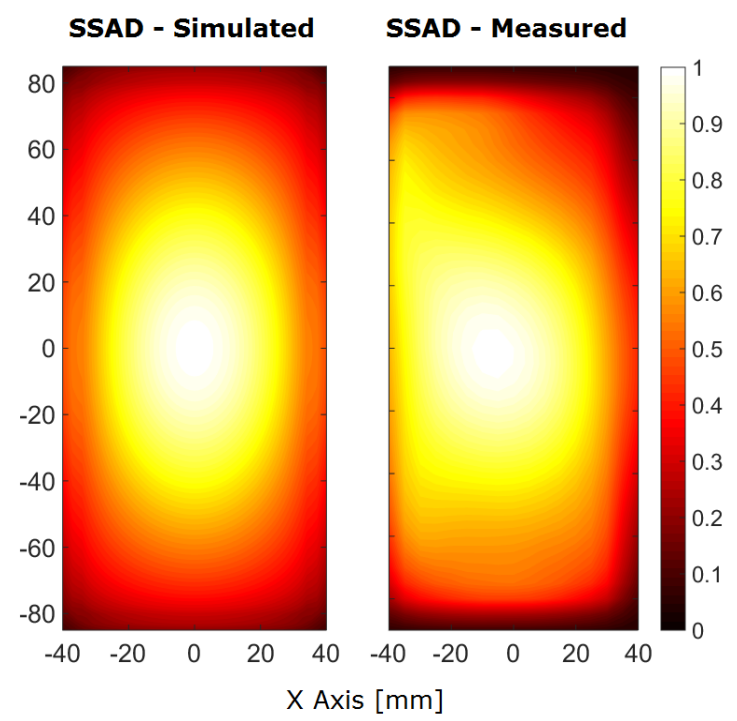

Fig. 8. Simulated and measured 1g-averaged SAR for the MRI Yagi (left) and SSAD (right) at the $x z$-plane placed $3 \mathrm{~mm}$ away from the phantom bottom. All measurements and simulations are normalized to the maximum local SAR obtained for the SSAD. 
propagation and also reduce the mutual coupling between elements when used in arrays. Directors, placed coplanar and separated a distance between $\lambda / 10$ and $\lambda / 8$, create something similar to a passive metasurface [13]. Metamaterial-like structures have already proven to be useful to increase $\mathrm{H}$-field around the sample surface [14] and to improve decoupling between array elements [15]. We obtained here similar results regarding $\mathrm{H}$-field increase and decoupling, but most importantly, we show that these kind of structures can also be used to greatly reduce SAR local maximums at locations close to the coil.

Adjusting the length of the active dipole is very important for optimal power delivery to the coil. Although a more compact coil (especially the SSAD) may be realized using a shorter dipole, this strategy will require a larger capacitor to be used for tuning. A larger current will then flow through the capacitor, which increases resistive loss. Therefore, the tuning capacitor has to be as low as possible while still allowing the retuning of the coil for different load conditions. In the examples studied here, this led to a slightly different length for the dipole arms of the SSAD and the MRI Yagi (46 and 49 $\mathrm{mm}$ respectively). Doing so, both coils could be tuned with a capacitance lower than $4 \mathrm{pF}$.

The proposed antenna is useful as a transmit-receive or transmit-only coil, due to its higher transmit efficiency. $B_{1}{ }^{+}$ homogeneity, which is a very important metric for transmit coils, is not evaluated here. However, Fig. 3 provides some indication that the homogeneity of the proposed design is similar to that of the SSAD for deep locations $(>2 \mathrm{~cm})$. Note that the frequency of operation might vary slightly in different 7T scanners (within a few tens of $\mathrm{kHz}$ ), but no significant changes are expected due to that variation.

\section{CONCLUSION}

A new MRI radiative coil using parasitic scatterers is proposed and compared to an SSAD of the same size. The proposed coil shows a $25 \%$ improvement on the ratio $B_{1}{ }^{+}$ $/ \sqrt{S A R}_{\mathrm{MAX}}$ at the sample depth of $15 \mathrm{~cm}$ when compared to the SSAD. Moreover, the use of parasitic scatterers in a Yagi fashion (with reflectors and directors) facilitates a significant increase in the directivity of the coil, which reduces back propagation and coupling to neighboring elements in an array configuration. The reduced coupling and compact coil size makes this design very promising for the development of high density radiative arrays. In practice, the improved transmit efficiency implies that either the amplitude or the duration of the transmitted RF pulse can be reduced by an equivalent factor, which makes this coil design useful in cases where local SAR limits the amount of transmit power, and also for low flip angle acquisitions.

Moreover, it is noted that the realization of the MRI Yagi coil in this paper is kept simple to facilitate proof of concept and experimental validation. Many of the design parameters could be further optimized to yield even better performance, including the number of layers of reflectors and directors and even the dielectric material. For example, the design proposed here uses distilled water as high $\varepsilon_{r}$ dielectric material for simplicity. However, more exotic materials like Barium Titanate (BaTiO3) with $\varepsilon_{r}>300$ can be used to further reduce the size of the coil, and are good candidates to explore the fundamental limits in miniaturizing the proposed design.

One option for further increasing SNR is to utilize dipole antenna arrays as transmitters and loop arrays as receivers [16]. This is because such a setup combines the higher penetration depth of dipoles with the higher sensitivity of loop antennas for reception. The design concept proposed here has the potential of combining both arrays into one, for example by adding wires to connect the outer ends of some directors via PIN diodes, which can then convert the directors into loop antennas during the receiving phase. Designs based on coaxial Yagi loop antennas could also be useful for this purpose [17]. Therefore, one promising track for future work is to optimize the MRI Yagi coil concept from a system perspective, taking into account both the transmitting and receiving aspects of the MRI experiment.

\section{REFERENCES}

[1] A. G. Webb, "Dielectric materials in magnetic resonance," Concepts Magn. Reson. Part A, vol. 38A, no. 4, pp. 148-184, Jul. 2011.

[2] E. Moser, F. Stahlberg, M. E. Ladd, and S. Trattnig, "7T MR - from research to clinical applications?" NMR Biomed., vol. 25, no. 5, pp. 695716, May 2012.

[3] A. J. E. Raaijmakers, et al., "Design of a radiative surface coil array element at 7T: The single-side adapted dipole antenna," Magn. Reson. Med., vol. 66, no. 5, pp. 1488-1497, Nov. 2011.

[4] P. B. Roemer, et al., "The NMR phased array," Magn. Reson. Med., vol. 16, no. 2, pp. 192-225, Nov. 1990.

[5] A. Graessl, et al., "Modular 32-channel transceiver coil array for cardiac MRI at 7.0T," Magn. Reson. Med., vol. 72, no. 1, pp. 276-290, Jul. 2014.

[6] P. Angelidis, K. Vassiliadis, and G. D. Sergiadis, "Lowest mutual coupling between closely spaced loop antennas," IEEE Trans. Antennas Propag., vol. 39, no. 7, pp. 949-953, Jul. 1991.

[7] H. Yagi and S. Uda, "Projector of the sharpest beam of electric waves," Proc. Imperial Academy, vol. 2, no. 2, pp. 49-52, 1926.

[8] H. Yagi, "Beam transmission of ultra short waves," Proc. IRE, vol. 16, no. 6, pp. 715-740, 1928.

[9] B. K. Lau and J. B. Andersen, "Simple and efficient decoupling of compact arrays with parasitic scatterers," IEEE Trans. Antennas Propag., vol. 60, no. 2, pp. 464-472, Feb. 2012.

[10] X. Yan, et al., "Magnetic wall decoupling method for monopole coil array in ultrahigh field MRI: a feasibility test," Quant. Imaging. Med. Surg., vol. 4, no. 2, pp. 79-86, 2014.

[11] A. J. E, Raaijmakers, et al. "The fractionated dipole antenna: A new antenna for body imaging at 7 tesla," Magn. Reson. Med., vol. 75, no. 3, pp. 1366-1374, Mar. 2016.

[12] J. T. Vaughan and J. R. Griffiths, Eds., RF coils for MRI. UK: John Wiley and Sons, 2012.

[13] B. O. Zhu and Y. Feng, "Passive metasurface for reflectionless and arbitary control of electromagnetic wave transmission," IEEE Trans. Antennas Propag., vol. 63, no. 12, pp. 5500-5511, Dec. 2015.

[14] M. S. Khennouche, F. Gadot, B. Belier, and A. de Lustrac, "Different configurations of metamaterials coupled with an RF coil for MRI applications," Appl. Phys. A, vol. 109, no. 4, pp. 1059-1063, 2012.

[15] A. A. Hurshkainen, et al., "Element decoupling of 7T dipole body arrays by EBG metasurface structures: experimental verification," J. Magn. Reson., vol. 269, pp. 87-96, Aug. 2016.M. A. Ertürk, et al., "A 16channel combined loop-dipole transceiver array for 7 tesla body MRI," Magn. Reson. Med., Feb. 2016.

[16] M. A. Ertürk, et al., "A 16-channel combined loop-dipole transceiver array for 7 Tesla body MRI". Magn. Reson. Med., vol. 77, pp. 884-894, Jan. 2017.

[17] A. Shoamanesh and L. Shafai, "Properties of coaxial Yagi loop arrays," IEEE Trans. Antennas Propag, vol. 26, no. 4, pp. 547-550, Jul 1978. 